\title{
Topographical distribution of trigeminal receptor expression in the nasal cavity*
}

\author{
Sophia C. Poletti1,2, Jacqueline Hausold ${ }^{1,3}$, Andreas Herrmann ${ }^{4}$, Martin Witt ${ }^{5}$, \\ Thomas Hummel ${ }^{1}$ \\ Rhinology 57: 2, 147 - 152, 2019 \\ https://doi.org/10.4193/Rhin18.181 \\ 'Smell and Taste Clinic, Department of Otorhinolaryngology, TU Dresden, Dresden, Germany \\ *Received for publication: \\ 2 Department of Otorhinolaryngology, Head and Neck Surgery, University Hospital Tuebingen, Tuebingen, Germany \\ August 13, 2018 \\ 3 Department of Gynecology, TU Dresden, Dresden, Germany \\ Accepted: October 26, 2018 \\ ${ }^{4}$ Department of Neurology, TU Dresden, Dresden, Germany \\ 5 Institute of Anatomy, Rostock University Medical Center, Rostock, Germany
}

Background: Topographical differences in trigeminal receptor distribution of the nasal cavity has been investigated so far indirectly using various agonists to stimulate receptors in different locations. However, polymodal activation of trigeminal receptors poses difficulties in such investigation. The aim of our study was to examine the distribution of trigeminal receptor mRNA expression using quantitative PCR.

Methodology: A prospective study was performed in 18 healthy volunteers. Mucosal biopsies were obtained from five different endonasal locations (covering the anterior, posterior and lateral nasal cavity) for receptor mRNA quantification by means of RTPCR.

Results: The highest overall level of RNA expression was found for TRPV1, followed by ACCN3, TRPA1 and TRPM8. Identical distribution pattern could be shown for each investigated area of the nasal cavity. Highest overall receptor density was found in the posterior septum due to high TRPV1 and ACCN3 receptor expression.

Conclusions: For the first time, we demonstrate a distinct trigeminal receptor RNA distribution pattern of the nasal mucosa. The highest overall distribution level was found in the posterior nose.

Key words: ACCN3, nose, trigeminal receptors, trigeminal sensitivity, TRPV1

\section{Introduction}

The trigeminal system responds to thermal, mechanical and chemical stimuli within the nasal and oral mucosa. Studies on anosmic humans and animals demonstrate the important role of the trigeminal somatosensory neurons in detecting a broad range of chemical irritants through the trigeminal system ${ }^{(1-4)}$. Contrary to previous assumptions of non-specific interactions between chemical composites and free trigeminal nerve ending $\mathrm{s}^{(5,6)}$, a variety of trigeminal receptors have been identified in recent years, indicating a ligand- receptor interaction similar to gustation and olfaction. Transient Receptor Potential (TRP) Channels, as the main representatives of nasal trigeminal receptors, are grouped by sequence similarity into several subfamilies. Representatives of three subfamilies were of greater interest in intranasal perception and therefore investigated in this study.
TRPV1, a vanilloid receptor highly sensitive to capsaicin ${ }^{(7)}$ and ethanol ${ }^{(8)}$, eliciting a burning pain sensation, TRPA1 receptors transmitting stinging and burning sensations following cinnamaldehyde and mustard oil activation ${ }^{(9,10)}$ and finally TRPM8, a cold- and menthol sensitive receptor ${ }^{(11)}$, inducing cooling sensations including sensations of airflow. In addition, the acidsensitive ion channel ACCN3 (or ASIC3) responds to reduction of extracellular $\mathrm{pH}$ and protons ${ }^{(12)}$.

Various studies investigated endonasal chemo-somatosensory perception of the trigeminal nerve using different methods. For example, in addition to electrophysiological ${ }^{(13-15)}$ and psychophysical ${ }^{(16)}$ measures in healthy humans, trigeminal sensitivity has also been investigated in patients with chronic rhinosinusitis and the effect of surgery ${ }^{(17)}$. These studies show a distinct mucosal distribution of trigeminal perception depending on the sti- 
mulus used. But predominantly a higher sensitivity for chemical stimuli was found at the anterior portion of the nose suggesting a sentinel function of the respiratory system. In these studies, however, different trigeminal agonists were used to activate the trigeminal system in various locations, aiming to indirectly show the trigeminal receptor distribution of the nasal cavity. To our knowledge, no direct proof of receptor distribution was attempted. Hence, the aim of our study was to investigate topographical differences in endonasal trigeminal receptor distribution based on quantification of receptor mRNA using RT-PCR.

\section{Material and Methods}

\section{Participants}

A prospective study was conducted at the Smell and Taste Clinic/ Department of Otolaryngology of the "Technische Universität" (TU) Dresden. The study was performed according to the Declaration of Helsinki and approved by the Ethics Committee of the Medical Faculty of the TU Dresden (application number: EK 88052006).

A total of 18 participants ( 7 females, 11 males; mean age: 37.9 years; SD: 14) scheduled for septorhinoplasty at the Department of Otolaryngology took part in the study. Only participants with a normal sense of smell assessed by the Sniffin' Sticks Screening test were included in the study. Further, nasal endoscopy was performed in all participants to rule out any endonasal diseases such as polyps or any form of chronic rhinosinusitis, which posed an exclusion criterion. Additionally, the following exclusion criteria applied: neurological diseases, any systemic diseases associated with smell disorders like chronic renal failure or thyroid disorders, participants with any type of smell impairment, allergic rhinitis, alcohol or drug abuse and those with systemic diseases relevant to the nose such as granulomatosis with polyangiitis.

\section{Assessment of olfactory function}

The "Sniffin' Sticks" Screening test ${ }^{(18,19)}$ (Burghart Messtechnick $\mathrm{GmbH}$, Wedel, Germany) was based on the identification of 12 common odors presented in felt- tip pen like odor dispensers. Odors were presented to the participants for 2 seconds at about $2 \mathrm{~cm}$ distance from the nostril. With each Sniffin' Stick presentation, participants were asked to identify the odor from a list of four descriptors in a forced choice procedure. Identifying 10 or more odors out of 12 was considered normosmic. However, this test does not allow any further differentiation of smell impairment in participants scoring lower than 10 points.

\section{Endonasal biopsy}

In accordance to a study from Scheibe et al. ${ }^{(20)}$ five areas in the nasal cavity were chosen for mucosal biopsy, namely the anterior wall of the sphenoid sinus (area 1), the anterior septum (2), the posterior septum (3), the insertion of the inferior turbinate

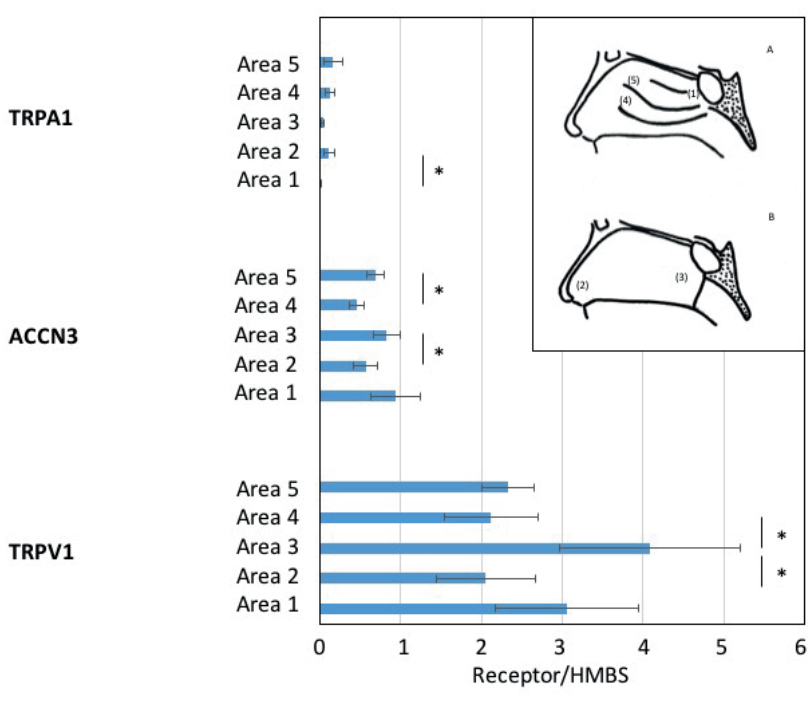

Figure 1. Nasal picture: (A) illustrates the lateral wall of the nasal cavity and (B) the nasal septum with all 5 locations mucosal biopsies were taken from: (1) anterior wall of the sphenoid sinus, (2) anterior septum, (3) posterior septum, (4) insertion of the inferior turbinate, (5) insertion of the middle turbinate. The graphs show TRPA1, ACCN3 and TRPV1 receptor RNA expression (in relation to the expression of the housekeeping gene HMBS) in different endonasal areas as demonstrates in the nasal picture. * indicates significant differences in RNA expression between sites.

(4) and the insertion of the middle turbinate (5). All biopsy locations are illustrated in Figure 1. The reason for this choice of locations was to cover the anterior, posterior, medial and lateral parts of the nasal cavity to investigate possible topographical differences of trigeminal receptor distribution. Biopsies were taken intraoperatively from patients undergoing septorhinoplasty for aesthetic or functional reasons. Before any local anaesthetics or any decongesting agent was applied to the nasal cavity, biopsies were randomly taken from both sides using a Blakesley forceps. Immediate transfer of the tissue samples to RNAlater containing RNAse free tubes (QIAGEN, Hilden, Germany, 2ml tubes) was guaranteed and preserved at $4^{\circ} \mathrm{C}$ for a maximum of 48 hours.

\section{Receptors of trigeminal perception}

From the Transient Receptor Potential Channels, three subfamilies are of greater interest in intranasal trigeminal perception (see introduction). Therefore, we used TRPV1, TRPA1 and TRPM8 to detect endonasal RNA expression by means of RT-PCR as described below. Further, RNA quantification was investigated for the acid sensing ion channel ACCN3 which plays an important role in the perception of painful und mechanical stimuli.

Quantitation of TRPV1, TRPA1, TRPM8 and ACCN3 mRNA by RT-PCR: RNA isolation and reverse transcription 
Table 1. Primer sequences of TRPM8 (Transient receptor potential cation channel subfamily M member 8) and HMBS (Hydroxymethyl-bilane synthase) ordered from Eurofins MWG/ Operon.

\begin{tabular}{|c|c|c|c|c|}
\hline Gene & Sequences & Accession number & length & $\operatorname{Tm}\left({ }^{\circ} \mathrm{C}\right)$ \\
\hline $\begin{array}{l}\text { TRPM8 (Transient receptor potential cation } \\
\text { channel subfamily M member } 8 \text { ) }\end{array}$ & $\begin{array}{c}\text { 5'-TCA AGC AAA TTT TAA GAA- 3' } \\
3^{\prime} \text {-CAA AGG CGT CGG TAG GAA AT- 5' }\end{array}$ & NM_024080.4 & $176 \mathrm{bp}$ & 81,0 \\
\hline HMBS (Hydroxymethyl-bilane synthase) & $\begin{array}{l}5^{\prime}-\mathrm{TCG} \text { GGG AAA CCT CAA CAC C- } 3^{\prime} \\
3^{\prime}-\text { CCT GGC CCA CAG CAT ACA T- } 5^{\prime}\end{array}$ & NM_000190.3 & $154 \mathrm{bp}$ & 86,0 \\
\hline
\end{tabular}

Table 2. Primer sequences of TRPA1 (transient receptor potential cation channel, subfamily A, member 1), TRPV1 (transient receptor potential cation channel, subfamily V, member 1) and ACCN3 (amiloride-sensitive cation channel 3) ordered from QIAGEN.

\begin{tabular}{|c|c|c|c|}
\hline Gene & Accession number & length & $\operatorname{Tm}\left({ }^{\circ} \mathrm{C}\right)$ \\
\hline TRPA1 (transient receptor potential cation channel, subfamily A, member 1 ) & NM_007332.2 & $136 \mathrm{bp}$ & 83 \\
\hline TRPV1 (transient receptor potential cation channel, subfamily V, member 1) & NM_018727.5 & 92 bp & 80,4 \\
\hline ACCN3 (amiloride-sensitive cation channel 3) & NM_004769 & $112 \mathrm{bp}$ & 80 \\
\hline
\end{tabular}

Total RNA was isolated using the optimized phenol guanidinium isothiocyanate extraction with the commercially available QIAGEN kit and in accordance with the manufacturer's instructions („RNeasy Lipid Tissue Mini Kit“).

Total RNA concentrations were determined by absorbance measurements at $260 \mathrm{~nm}$. Subsequently, $1 \mu \mathrm{g}$ total RNA was reversetranscribed using RevertAidTM First Strand cDNA Synthesis-Kit in accordance with the given instructions (Fermentas $\mathrm{GmbH}$, St.Leon-Roth, Germany). Quantitative PCR of CDNA was also done in line with the manufacturer's instructions using Brillant ${ }^{\circledR}$ II SYBR ${ }^{\circledR}$ Green QRT-PCR Master Mix Kit (QIAGEN) on a Thermal Cycler with an integrated micro volume fluorimeter (Techne TC 512, Bibby Scientific Limited, Beacon Road, Stone, Staffordshire ST15 OSA, UK). The detailed methodology is described elsewhere ${ }^{(21,22)}$.

The PCR was performed using HMBS (Hydroxymethylbilan synthase) as the housekeeping gene and gene-specific primers synthesized according to published CDNA sequences of TRPV1, ACCN3, TRPA1, TRPM8 (Table 1, 2).

Reactions were run under the following cycling conditions: $94^{\circ} \mathrm{C}$ for 2 min (initial activation) succeeded from 35 cycles of amplification. Each cycle included $30 \mathrm{~s}$ denaturation at $95^{\circ} \mathrm{C}$, $30 \mathrm{~s}$ annealing at $55^{\circ} \mathrm{C}$ and $30 \mathrm{~s}$ of synthesis at $72^{\circ} \mathrm{C}$. Amplified PCR products were visualized through electrophoresis in a $1.5 \%$ agarose gel (Biozym LE, Hessisch-Oldendorf / Germany) containing ethidium bromide at room temperature and at an electrical field of $200 \mathrm{~V}$.

\section{Statistical analysis}

Data analyses were done by means of SPSS (Statistical Package for Social Sciences, version 25.0, SPSS Inc., Chicago, IL, USA) using analyses of variance (ANOVA) for repeated measures ("within-subject-factor" [endonasal locations]). The t-tests for paired samples were used to explore differences in receptor mRNA expression between different endonasal locations in case the main effect was significant. The level of significance was set at $\mathrm{p}<0.05$.

\section{Results}

\section{Overall receptor distribution}

For each of the 5 biopsy sites the highest rate for detection was 16 out of 18 participants for TRPV1, 16 of 18 for ACCN3, 14 of 18 for TRPA 1 and 12 of 18 for TRPM8. The highest overall RNA expression in the nasal mucosa was found for the TRPV1 receptor, followed by ACCN3, TRPA1 and TRPM8.

\section{Receptor distribution in relation to the location}

At the anterior sphenoid sinus wall (area 1) TRPV1 had the highest RNA expression amongst all receptors (mean: 3.05; SD: 0.88; range: $0.58-11.71$ ), followed by the expression of ACCN3 (mean: 0.94; SD: 0.31; range: 0.02-3.76). Very low RNA expression was found in TRPA 1 (mean: 0.016; SD: 0.005; range: 0.0002-0.07) and TRPM8 (mean: 0.0008; SD: 0.0006; range: 0.00003-0.005). At the anterior septum (area 2) as in area 1 TRPV1 had the highest RNA expression (mean: 2.05; SD: 0.62; range: 0.22-8.57) proceeded from ACCN3 (mean: 0.57; SD: 0.15; range: 0.09-1.74), while TRPA 1 (mean: 0.11 ; SD: 0.07; range: 0.006-0.78) and TRPM8 (mean: 0.003; SD: 0.002; range: 0.00003-0.03) were low to very low in RNA expression.

At the posterior septum (area 3 ) there is a similar receptor distribution as in area 1 and 2 with the highest RNA expression found for TRPV1 (mean: 4.08; SD: 1.12; range: 0.95-17.39) followed by ACCN3 (0.46; SD: 0.09; range: 0.09-1.12) and a very low expression of TRPA 1 (0.04; SD: 0.01; range: 0.001-0.16) and TRPM8 (0.0013; SD: 0.0006; range: 0.0001-0.007).

At the insertion of the inferior and the middle turbinate (areas 
4 and 5), again TRPV1 RNA (it: mean: 2.12; SD: 0.58; range:

0.14-8.11; mt: mean: 2.33; SD: 0.32; range: 0.74-4.82) was mainly expressed, followed by ACCN3 (it: mean: 0.46; SD: 0.09; range: 0.09-1.12; mt: mean: 0.69; SD: 0.40; range: 0.13-1.72), TRPA1 (it: mean: 0.13 ; SD: 0.06; range: 0.005-0.63; mt: mean: 0.17; SD: 0.12; range: 0.002-1.73) and TRPM8 (it: mean: 0.0007; SD: 0.0003; range: 0.00006-0.004; mt: mean: 0.0008; SD: 0.0003; range: 0.00002-0.004).

Taken together, TRPV1 was found to have consistently the highest RNA expression in all areas, followed by ACCN3, TRPA1 and TRPM8.

\section{Receptor types and their distribution}

TRPV1 had the highest expression at the posterior septum (area 3), followed by the insertion of the middle turbinate (area 5), the anterior sphenoid sinus wall (area 1), the insertion of the inferior turbinate (area 4) and the anterior septum (area 2). There was a significantly higher RNA expression at the posterior septum as compared to the anterior septum ( $\mathrm{t}(13)=-2,913, \mathrm{p}=0.012$ ) and the insertion of the inferior turbinate $(t(12)=2,791, p=0.016)$. No significant differences emerged between the other areas (all ps > 0.05).

Overall, ACCN3 was less expressed in all investigated areas than TRPV1. The highest expression was registered at the anterior sphenoid sinus wall (area 1), followed by the posterior septum (area 3), the insertion of the middle turbinate (area 5), the anterior septum (area 2) and the inferior turbinate (area 4). As with TRPV1, a significantly higher RNA expression could be shown at the posterior septum as compared to the anterior septum ( $t$ (12) $=-2.68, p=0.02$ ). Further, significantly higher ACCN3 RNA could be detected at the insertion of the middle turbinate as compared to the inferior turbinate $(t(12)=-2.21, p=0.047$ ).

TRPA 1 had the third lowest overall RNA expression in the nasal mucosa with the highest expression, contrary to the other two receptors, located at the antero- inferior portions of nasal cavity, namely the insertion of the inferior turbinate and the anterior septum. In contrast, the lowest expression was found in the postero- superior areas of the nose, like the posterior septum followed by the insertion of the middle turbinate and the sphenoid sinus. In line with this distribution pattern, a significantly higher RNA expression was found at the anterior septum as compared to the anterior sphenoid sinus wall ( $t(9)=-2,936, p=0.017$ ). Result of TRPV1, ACCN3 and TRPA1 are demonstrated in Figure 1. In general, very low expression of the TRPM8 RNA was found in all areas. However, looking at their distribution, a predominantly medial mucosal wall distribution emerged with the highest concentrations found at the anterior sphenoid sinus wall, the posterior septum and the anterior septum. Whereas the lateral wall with the insertion of the middle and the inferior turbinate was very low in TRPM8 RNA expression. However, no significant differences emerged between the areas (all ps $>0.05$ ).

\section{Discussion}

The results of this study demonstrate that 1) the highest overall RNA expression in the nasal mucosa was found for TRPV1, followed by ACCN3, TRPA1 and TRPM8. Further, 2) in each area of the nose TRPV1 was found to have the highest RNA expression. Interestingly, 3) identical patterns of distribution were found in each investigated nasal area. Looking at the receptor expression on an individual level, we could show that 4) TRPV1 was primarily expressed in the posterior parts of the nose, namely the posterior septum, with a significantly higher expression as compared to the anterior parts like the anterior septum and the insertion of the inferior turbinate. 5) Likewise, ACCN3 was mainly expressed in the posterior portions with a significantly higher expression seen in the posterior septum as compared to the anterior septum. 6) Consequently, highest receptor density was found in the posterior septum. In contrast to TRPV1 and ACCN3, 7) TRPA 1 was mainly expressed in the antero- inferior portions of the nose with a significantly higher RNA expression in the anterior septum as compared to the anterior sphenoid wall. 8) Due to limited TRPM8 mRNA detectability with borderline values, no meaningful interpretation could be drawn regarding its topographical distribution.

Several studies looked at the topographical differences in trigeminal perception of the nasal cavity using various methodological approaches. Scheibe et al. used negative mucosa potential (NMPs) recordings - an electrophysiological measure of trigeminal activity at the level of the respiratory epithelium - in response to $\mathrm{CO}_{2}$ stimulation to show highest sensitivity at the anterior part of the endonasal cavity ${ }^{(15)}$. Using recordings of chemosensory event-related potentials (ERP) in response to $\mathrm{CO}_{2}$ stimulation, Frasnelli et al. showed, in line with the above- mentioned studies, the highest sensitivity at the anterior portion of the nasal cavity. This view, however, is questioned by work from Melzner et al. ${ }^{(23)}$ indicating that the posterior part of the nasal cavity exhibits higher trigeminal sensitivity at threshold level than anterior portions.

We looked at the topographical distribution of selected trigeminal receptors by means of receptor RNA quantification using RT-PCR. Considering that $\mathrm{CO}_{2}$ mainly activates ACCN3 receptors, our study shows, contrary to most results discussed above that would favour a high ACCN3 expression in the anterior portions, a significantly higher ACCN3 expression in the posterior as compared to the anterior septum. Meusel et al. also demonstrate significantly higher NMP responses to $\mathrm{CO}_{2}$ at the anterior parts of the nose ${ }^{(24)}$. However, NMP amplitudes in response to ethanol, a substance mainly activating TRPV1 receptors, were found to be largest at the posterior septum, which confirms on a functional level our findings of a significantly higher TRPV1 RNA expression in the posterior septum as compared to the anterior portions of the nose. However, polymodal activation of trigeminal receptors, indicating a receptor sensitivity to multiple stimuli ${ }^{(25,26)}$, 
poses an additional challenge to investigate topographical distributions of receptors. In this case, the most reliable method to study endonasal distribution of trigeminal receptors would be direct proof of receptor RNA from various endonasal locations, as done in our study.

Further, with the highest overall RNA expression found in the posterior nose, the potential importance of the posterior region for airway protection might have been underestimated and contradicts the so far generally accepted idea of the anterior nose as a sole protector of the airway.

Due to the invasiveness of tissue sampling, a limited number of subjects was recruited. Along with potential methodological barriers, this led to a limited RNA detectability, at least for some of the investigated receptors. However, even if the RNA concentrations might be underestimated, the similar distribution pattern of the receptors across all mucosal areas seems to represent a reliable picture of the endonasal trigeminal receptor arrangement. In this case, TRPV1 and ACCN3, showing the highest expression amongst the investigated receptors, seem to play a major role in endonasal trigeminal perception, as seen for pain perception in animals lacking the TRPV1 channel ${ }^{(27,28)}$. TRPM8 showed very low RNA expression in the mucosal biopsies as compared to TRPV1 and ACCN3. TRPM8 receptors seem to be located in the sub-epithelium or deeper glandular regions, while TRPV1 was detected rather in the peripheral epithelium ${ }^{(29)}$ which could explain the differences in RNA detectability. Work by Scheibe et al. ${ }^{(30)}$ indicates that decreased endonasal trigeminal sensitivity may result in a feeling of nasal congestion which in turn may lead to surgical procedures. Considering that all participants from our study underwent septorhinoplasty for aesthetic but also functional reasons, we have to take into account that the low concentrations of TRPM8- a receptor which was reported to play an important role for airstream detectability in nasal breathing ${ }^{(31,32)}$ - might be underrepresented in the present population. Whether the methodological limitations, the selection bias or others are accountable for the very low detectability of TRPM8 in particular remains unclear and should be further investigated.

Finally, in this study, we wanted to investigate the topographical distribution of trigeminal receptors in the nasal cavity and the feasibility of this study using RT-PCR to detect various receptor mRNA. No psychophysical testing was performed to correlate with the receptor expression. However, future studies are needed to investigate the correlation between trigeminal function and receptor distribution in healthy and diseased participants.

\section{Conclusions}

In summary, we demonstrated for the first time a distinct distribution of trigeminal receptor RNA in the nasal mucosa using quantitative RT-PCR. While the present literature indicates higher trigeminal sensitivity in the anterior portions of the nose, our study shows a rather posteriorly dominated mRNA expression for the investigated receptors, with the highest expression found for TRPV1 and ACCN3.

\section{Acknowledgements}

This work was supported by a grant from the 'Deutsche Forschungsgemeinschaft' to TH (DFG HU441/18-1).

\section{Authorship contribution}

TH: Contributed to study conception and design, analysis and interpretation of the data, drafting and critical revision of the manuscript; JH: Data acquisition and critical revision of the manuscript; $\mathrm{AH}$ : Contributed to study conception and critical revision of the manuscript; MW: Contributed to study conception, analysis and interpretation of the data and critical revision of the manuscript; SCP: Contributed to analysis and interpretation of the data, drafting and revising of the manuscript.

\section{Conflict of interest}

The authors declare no conflict of interest.

\section{References}

1. Doty RL. Intranasal trigeminal detection of chemical vapors by humans. Physiol Behav 1975; 14(6):855-9.

2. Doty RL, Brugger WE, Jurs PC, Orndorff MA, Snyder PJ, Lowry LD. Intranasal trigeminal stimulation from odorous volatiles: Psychometric responses from anosmic and normal humans. Physiol Behav 1978; 20:175-85.

3. Silver WL, Moulton DG. Chemosensitivity of rat nasal trigeminal receptors. Physiol Behav 1982; 28:927-31.

4. Mason JR, Silver WL. Trigeminally mediated odor aversions in starlings. Brain Res 1983; 269:196-9.

5. Radil T, Wysocki CJ. Spatiotemporal masking in pure olfaction. Ann N Y Acad Sci 1998; 855:641-4.

6. Cain WS, Murphy CL. Interaction between chemoreceptive modalities of odour and irritation. Nature 1980; 284:255-7.

7. Caterina MJ, Schumacher MA, Tominaga M, Rosen TA, Levine JD, Julius D. The capsaicin receptor: a heat-activated ion channel in the pain pathway. Nature 1997; 389:816-24.

8. Nicoletti P, Trevisani M, Manconi M, Gatti R, De Siena G, Zagli G, et al. Ethanol causes neurogenic vasodilation by TRPV1 activation and CGRP release in the trigeminovascular system of the guinea pig. Cephalalgia 2008; 28:9-17.

9. Jordt S-E, Bautista DM, Chuang $\mathrm{H}-\mathrm{H}$, McKemy DD, Zygmunt PM, Högestätt ED, et al. Mustard oils and cannabinoids excite sensory nerve fibres through the TRP channel ANKTM1. Nature 2004; 427:260-5.

10. Bandell M, Story GM, Hwang SW, Viswanath $V$, Eid SR, Petrus MJ, et al. Noxious cold ion channel TRPA1 is activated by pungent compounds and bradykinin. Neuron 2004; 41:849-57.

11. McKemy DD, Neuhausser WM, Julius D. Identification of a cold receptor reveals a general role for TRP channels in thermosensation. Nature 2002; 416:52-8.

12. Waldmann R, Champigny G, Bassilana F, Heurteaux C, Lazdunski M. A proton-gated cation channel involved in acid-sensing. Nature 1997;386:173-7.

13. Hummel T, Schiessl C, Wendler J, Kobal G. 
Peripheral electrophysiological responses decrease in response to repetitive painful stimulation of the human nasal mucosa. Neurosci Lett 1996;212:37-40.

14. Frasnelli J, Heilmann S, Hummel T. Responsiveness of human nasal mucosa to trigeminal stimuli depends on the site of stimulation. Neurosci Lett. 2004; 362:65-9.

15. Scheibe M, Zahnert $T$, Hummel T. Topographical differences in the trigeminal sensitivity of the human nasal mucosa. Neuroreport 2006; 17:1417-20.

16. Wrobel BB, Bien AG, Holbrook EH, Meyer GE, Bratney NA, Meza J, et al. Decreased nasal mucosal sensitivity in older subjects. Am J Rhinol 2006; 20:364-8

17. Poletti SC, Cuevas M, Weile S, Hummel T. Trigeminal sensitivity in chronic rhinosinusitis: Topographical differences and the effect of surgery. Rhinology 2017; 55: 70-74.

18. Hummel T, Sekinger B, Wolf SR, Pauli E, Kobal G. "Sniffin" sticks': olfactory performance assessed by the combined testing of odor identification, odor discrimination and olfactory threshold. Chem Senses 1997; 22:39-52.

19. Kobal G, Hummel T, Sekinger B, Barz S, Roscher S, Wolf S. "Sniffin" Sticks': Screening of olfactory performance. Rhinology 1996; 34:222-6

20. Scheibe $M$, Schmidt $A$, Hummel $T$. Investigation of the topographical differences in somatosensory sensitivity of the human nasal mucosa. Rhinology 2012:50:290-3.

21. Wittwer CT, Ririe KM, Andrew R V, David DA,
Gundry RA, Balis UJ. The LightCycler(TM): A microvolume multisample fluorimeter with rapid temperature control. Biotechniques 1997;22:176-81.

22. Wittwer CT, Herrmann MG, Moss AA Rasmussen RP. Continuous Fluorescence Monitoring of Rapid Cycle DNA Amplification. Biotechniques 2013; 54:31420.

23. Melzner J, Bitter T, Guntinas-Lichius O, Gottschall R, Walther M, Gudziol H. Comparison of the orthonasal and retronasal detection thresholds for carbon dioxide in humans. Chem Senses 2011; 36:435-41.

24. Meusel T, Negoias S, Scheibe M, Hummel T. Topographical differences in distribution and responsiveness of trigeminal sensitivity within the human nasal mucosa. Pain 2010; 151:516-21.

25. Klein AH, Carstens MI, Zanotto KL, Sawyer CM, Ivanov M, Cheung S, et al. Self- and cross-desensitization of oral irritation by menthol and cinnamaldehyde (CA) via peripheral interactions at trigeminal sensory neurons. Chem Senses 2011; 36:199-208.

26. Zheng J. Molecular Mechanism of TRP Channels. Compr Physiol 2013; 3:221-42.

27. Caterina MJ, Leffler A, Malmberg AB, Martin WJ, Trafton J, Petersen-Zeitz KR, et al. Impaired nociception and pain sensation in mice lacking the capsaicin receptor. Science 2000; 288:306-13.

28. Davis JB, Gray J, Gunthorpe MJ, Hatcher JP Davey PT, Overend P, et al. Vanilloid receptor-1 is essential for inflammatory thermal hyperalgesia. Nature 2000; 405:183-7.
29. Keh SM, Facer P, Yehia A, Sandhu G, Saleh HA, Anand P. The menthol and cold sensation receptor TRPM8 in normal human nasal mucosa and rhinitis. Rhinology 2011;49:11.

30. Scheibe M, Schulze S, Mueller CA, Schuster $B$, Hummel T. Intranasal trigeminal sensitivity: measurements before and after nasal surgery. Eur Arch Otorhinolaryngol 2014; 271:87-92.

31. Zhao K, Blacker K, Luo Y, Bryant B, Jiang J. Perceiving nasal patency through mucosal cooling rather than air temperature or nasal resistance. PLoS One 2011; 6:e24618.

32. Zhao K, Jiang J, Blacker K, Lyman B, Dalton P, Cowart BJ, et al. Regional peak mucosal cooling predicts the perception of nasal patency. Laryngoscope 2014; 124:589-95.

Thomas Hummel, MD

Smell and Taste Clinic

Department of Otorhinolaryngology

TU Dresden

Fetscherstrasse 74

01307 Dresden

Germany

Tel: +49-351-458-4189

Fax +49-351-458-4326

E-mail:

thummel@mail.zih.tu-dresden.de 